Applied and

NISTIR 5848

Computational

Mathematics

Division

Computing and Applied Mathematics Laboratory

\title{
On the Notion of a $\xi$-Vector and a Stress Tensor For a General Class of Anisotropic Diffuse Interface Models
}

\author{
A. A. Wheeler and G. B. McFadden,
}

April 1996

\section{U.S. DEPARTMENT OF COMMERCE}

Technology Administration

National Institute of Standards and Technology

Gaithersburg, MD 20899

$Q C$

100

.456

N0. 5848 



\title{
NISTIR 5848
}

\section{On the Notion for a $\xi$-Vector and a Stress Tensor For a General Class of Anisotropic Diffuse Interface Models}

\author{
A. A. Wheeler \\ G. B. McFadden
}

U.S. DEPARTMENT OF COMMERCE

Technology Administration National Institute of Standards and Technology

Applied and Computational Mathematics Division Computing and Applied Mathematics Laboratory Gaithersburg, MD 20899

April 1996

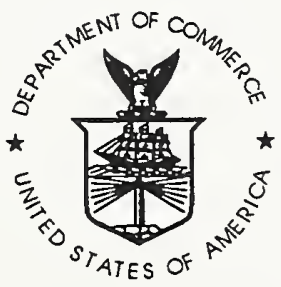

U.S. DEPARTMENT OF COMMERCE Michael Kantor, Secretary

TECHNOLOGY ADMINISTRATION

Mary L. Good, Under Secretary for Technology

NATIONAL INSTITUTE OF STANDARDS

AND TECHNOLOGY

Arati Prabhakar, Director 



\title{
On the Notion of a $\xi$-vector and a Stress Tensor For a General Class of Anisotropic Diffuse Interface Models
}

\author{
A. A. Wheeler \\ Faculty of Mathematical Studies \\ University of Southampton \\ Highfield, Southampton SO17 1BJ \\ $\mathrm{UK}$ \\ $\&$ \\ G. B. McFadden \\ National Institute of Standards and Technology \\ Gaithersburg, MD 20899 \\ USA
}

\begin{abstract}
We show that under equilibrium conditions, an anisotropic phase-field model of a diffuse interface leads to the notion of a divergence-free tensor, whose components are negligible away from the interface. Near the interface, it plays the rôle of a stress tensor, and involves the phase-field generalisation of the Cahn-Hoffman $\xi$-vector. We show that this tensor may be used to derive the equilibrium conditions for edges in interfaces. We then extend these ideas from the phase-field model to a general class of multiple-order-parameter models. In this broader context we demonstrate that it is also possible to construct a $\xi$-vector, and we use it to derive the three-dimensional form of the Gibbs-Thomson equation in the sharp interface limit. We derive the associated stress tensor for the multiple-order-parameter case, and show that it also leads to the appropriate equilibrium conditions for edges in interfaces and multiple junctions in the sharp-interface limit. This approach shows that both multiple-order-parameter models and less physically-based phase-field models share a common framework, the former providing a generalisation of the notion of the $\xi$-vector for phase-field models as well as providing a connection to the classical $\xi$-vector theory for a sharp interface. For the special case of a phase-field model with non-convex surface energy, we show that edges can be represented by weak shocks in which the spatial derivatives of the phase field are not continuous, and we derive the associated jump conditions. In all the situations considered the notion of the $\xi$-vector and the stress tensor play a central rôle.
\end{abstract}




\section{Introduction}

In many continuum theories of phase change the interface separating distinct bulk phases is considered to be diffuse, with a finite thickness over which properties vary smoothly from one set of bulk values to another. Theories which treat inter-phase boundaries as diffuse interfaces with characteristic thicknesses arise naturally in the description of critical phenomena (Stanley 1971). Using an approach similar to that of the Model C of Halperin, Hohenberg, and Ma (1974), J. S. Langer (unpublished, 1978) developed a model which has become known as the phase-field model. In this type of model, an auxiliary variable, the phase field $\phi$, is appended to the usual set of thermodynamic variables in order to provide an explicit indication of the thermodynamic phase of a non-uniform system at each point in space (Langer 1986, Caginalp \& Fife 1986). The free energy of the system, $\mathcal{F}$, includes contributions from the bulk energies, described by the free energy density $f(\phi, T)$, appropriate to the regions far from interphase boundaries, and terms involving the square of the gradient of the order parameter, which contribute to the energy in the transition regions, i.e.,

$$
\mathcal{F}=\int\left\{f(\phi, T)+\frac{\eta^{2}}{2}|\nabla \phi|^{2}\right\} d V
$$

where $\eta$ is a constant, and $T$ is the temperature. Dynamical equations for the evolution of the system can then be derived using variational arguments in the manner of irreversible thermodynamics (Penrose \& Fife 1990, Wang et al. 1993).

The governing equation for the evolution of the phase field is devised so that for small $\eta$ smooth but thin transition layers of width proportional to $\eta$ form between the phases, and the interface can be associated with a particular contour of the phase field, or more generally with the finite region over which the gradient of the phase field is significant. In the sharp-interface limit, $\eta \rightarrow 0$, in which the interface thickness tends to zero, it may be shown that the appropriate sharp-interface boundary conditions can be recovered (Caginalp 1989).

Phase-field models can be particularly useful for numerical computation in situations in which the interphase boundary is expected to be geometrically complicated. For example, during the dendritic growth of a pure material into its undercooled melt (Glicksman \& 
Marsh 1993), the generation and propagation of sidebranches along the primary branch of the dendrite leads to a wide range of length and time scales, and phase-field models have been employed to provide numerical tests of theories of tip selection (Wheeler et al. 1993, Karma and Rappel 1996). During dendritic growth an important rôle is played by the crystalline anisotropy of the growing solid phase, which selects the growth direction of the dendrite and determines the symmetry of the sidebranch structure. For a phase-field model to be of practical importance, it must include a description of the anisotropic nature of the crystal, through such effects as the dependence of the solid-liquid surface energy on the interface orientation with respect to the crystal lattice, and also the variation of the rate of attachment kinetics with interface orientation. Such anisotropic effects have been modeled by allowing the phenomenological phase-field parameters (such as the gradient energy coefficient, and the mobility coefficient for temporal relaxation) to depend on the orientation of the contours of the phase field (Kobayashi 1993). In this case the free energy of the system, in two dimensions, may be represented as

$$
\mathcal{F}=\int\left\{f(\phi, T)+\frac{\eta^{2}(\theta)}{2}|\nabla \phi|^{2}\right\} d V
$$

where $\tan (\theta)=\phi_{y} / \phi_{x}$. This approach successfully generalises the original isotropic phasefield methodology to allow the computation of dendrites with the proper qualitative behavior, and leads to the proper anisotropic version of the Gibbs-Thomson equation at the crystalmelt interface in the sharp interface limit (McFadden et al. 1993; Wheeler \& McFadden 1996). With this type of formulation, anisotropies with general symmetries can be treated using a single order parameter; for example, two-dimensional dendrites with six-fold symmetry can be computed using finite differences on a rectangular mesh (Murray et al. 1994; Warren and Boettinger 1995). On the other hand, with this approach the anisotropy is introduced in a somewhat ad hoc manner, and the connection with the underlying crystalline anisotropy is rather indirect.

An early attempt in the context of phase-field models to relate anisotropy to the crystal lattice is due to Caginalp and Fife (1986) who used methods from statistical mechanics applied to spins on a Bravais lattice to obtain a free-energy functional of the form

$$
\mathcal{F}=\int\left\{f(\phi, T)+\sum_{i=1}^{i=3} \frac{\eta_{i}^{2}}{2}\left(\frac{\partial \phi}{\partial x_{i}}\right)^{2}\right\} d V
$$


where $\eta_{i}$ are constant coefficients.

A conceptual shortcoming with phase-field models lies in the physical interpretation of the phase-field variable in the context of a solid/liquid phase transition to which they are commonly applied. In this setting the phase field does not provide a realistic description of the microscopic internal structure of the interface, and the utility of phase-field models rests in the recovery of the correct free-boundary problem in the sharp interface limit.

Phase-field models are one example of gradient energy models which date back to work by Rayleigh (1892) and van der Waals (1893), and are useful in a variety of contexts, including the description of superconductivity by Landau-Ginzburg theory (Ginzburg and Landau 1950), models of nonlinear elasticity (see, e.g., Carr et al. 1985), and the structure of fluid interfaces (see, e.g., Cahn and Hilliard 1958, Davis and Scriven 1982). In many cases gradient energy models can be viewed as mean field approximations to models that provide atomiclevel descriptions, such as in the Landau theory of a second-order phase transition (Landau 1937) or in density functional theories (see, e.g., Evans 1979 or Oxtoby 1991).

For a binary alloy with an atomic lattice with $N$ independent sublattices, $N$ concentrations are introduced which represent the concentration of one of the atomic species on each sublattice. The thermodynamic state of the system is described by an average concentration over the $N$ sublattices, together with $N-1$ order parameters, denoted by $X_{1}, X_{2}, \ldots, X_{N-1}$, that describe how atoms are distributed between the different sublattices. A gradient energy formulation is derived that gives rise to coupled evolution equations for the multiple order parameters. The resulting model can be regarded as a generalisation of the analogous CahnAllen model (Cahn \& Allen 1977, Allen \& Cahn 1979), for which a single order parameter suffices to describe the state of order on the two independent sites in a bcc crystal. Such a model involving a gradient energy formulation for three order parameters describing an fcc crystal was considered by Lai (1990) and Braun et al. (1996). In that situation with the concentration fixed the free energy functional has the form

$$
\mathcal{F}=\int\left\{f(X, Y, Z)+\frac{A}{2}\left(X_{x}^{2}+Y_{y}^{2}+Z_{z}^{2}\right)+\frac{B}{2}\left(X_{y}^{2}+X_{z}^{2}+Y_{x}^{2}+Y_{z}^{2}+Z_{x}^{2}+Z_{y}^{2}\right)\right\} d V
$$

where $A, B$ are positive constants which represent the nearest neighbour and second nearest neighbour interactions of atoms on the fcc lattice; $X, Y, Z$ are the corresponding order 
parameters and $f(X, Y, Z)$ is the bulk free energy.

In contrast to anisotropic phase-field models based on the free energy functional (2), where the form of surface energy anisotropy is completely specified through $\eta(\theta)$, in multipleorder-parameter models the surface energy anisotropy must in general be determined by solving the underlying Euler-Lagrange equations. This reflects the fact that the latter models inherit their surface energy anisotropy from the underlying lattice as well as provide a more detailed and realistic description of the structure of the interface than phase-field models.

The form of the surface energy anisotropy determines the equilibrium conditions at the interface. In the context of a sharp interface description, Hoffman and Cahn (Hoffman \& Cahn 1972, Cahn \& Hoffman 1974) developed a $\xi$-vector theory (briefly outlined below) which provides an elegant and compact device for describing the associated anisotropic form of the Gibbs-Thomson equation, the stresses acting in the interface, as well as treating the force balance at corners and edges that can form in the equilibrium shape when the surface energy anisotropy is sufficiently pronounced. Recently we showed that the $\xi$-vector can be applied to the above phase-field models and used it to show that the appropriate threedimensional Gibbs-Thomson equation is recovered in the sharp-interface limit (Wheeler \& McFadden 1996).

In this paper we consider a general class of anisotropic diffuse interface models which include, as special cases, the various forms of anisotropic phase-field models, and multipleorder-parameter models described above. We confine our attention to the case where, in the sharp interface limit, the surface energy $\gamma(\vec{n})$ is a differentiable function of the the unit normal vector to the interface (consideration of the nondifferentiable case is given by Taylor and Cahn 1996). We show that it is possible, in the sharp interface limit, to define a $\xi$ vector which is equivalent to that given by Hoffman and Cahn, and show that it plays a central rôle in the description of many aspects of the interface. We also show that we may associate a stress tensor with the interfacial region which we use to analyse stationary edges and line junctions. For the particular case of the phase-field model we show that edges are represented by weak shocks and we derive the corresponding jump conditions.

For clarity we first develop the theory for the particular case of a phase-field model in sections 2 and 3. Specifically, in section 2 we derive a stress tensor and use it to obtain the 
force acting in the interface in the sharp interface limit. In section 3 we use these results to consider the situation when the surface energy anisotropy is so large that edges form. In section 4 we consider the general multiple-order-parameter model and derive a $\xi$-vector, the corresponding three-dimensional form of the anisotropic Gibbs-Thomson equation in the sharp interface limit, and extend the results in the previous two sections to the multipleorder-parameter model.

\subsection{Cahn-Hoffman $\xi$-vector}

Hoffman and Cahn developed the $\xi$-vector to describe surface energy anisotropy in a first order phase transition represented by a sharp interface. The orientation of a surface element can characterised in terms of its local normal vector, $\vec{n}$, or equivalently by the spherical coordinates $\theta$ and $\phi$ that define the radial unit vector $\vec{r}(\theta, \phi)=\vec{n}$. If the surface energy is then written in the form $\gamma=\gamma(\theta, \phi)$, Hoffman and Cahn defined the $\xi$-vector by

$$
\vec{\xi}=\gamma \vec{r}+\gamma_{\theta} \vec{\theta}+\frac{\gamma_{\phi}}{\sin \theta} \vec{\phi}=\nabla[r \gamma(\theta, \phi)] .
$$

The unit vectors $\vec{\theta}$ and $\vec{\phi}$ are then tangent to the interface. In the isotropic case (constant $\gamma$ ), the $\xi$-vector thus reduces to the form $\vec{\xi}=\gamma \vec{n}$. More generally, the $\xi$-vector is in the direction of of the normal to the $1 / \gamma(\theta, \phi)$ plot defined by $r=1 / \gamma(\theta, \phi)$. Hoffman and Cahn showed that the interface satisfies the condition

$$
\nabla_{S} \cdot \vec{\xi}=-\Delta \dot{f}
$$

where $\nabla_{S}$. is the surface divergence on the interface and $\Delta f$ is the bulk free energy difference across the interface. The corresponding Gibbs-Thomson equation for an anisotropic surface energy may be written in the form

$$
T=T_{M}-\frac{T_{M}}{L} \nabla_{S} \cdot \vec{\xi}
$$

where $T$ is the local interface temperature, $T_{M}$ is the melting point, and $L$ is the latent heat of fusion per unit volume. They went on to show that the equilibrium shapes are given by $\vec{x}=\vec{\xi} / \Delta f$, i.e., the envelope of the $\xi$-vectors, which is equivalent to the Gibbs-Wulff construction (see, e.g., Taylor 1992). If the surface energy anisotropy is so pronounced that 
the $1 / \gamma$-plot is not convex, they showed that corners form in the equilibrium shape and, in two space dimensions, they demonstrated that the $\xi$-vector is continuous across corners. Further, they showed that the force per unit length, $\vec{f}$, acting on a line element in the interface in the direction of the unit vector $\vec{l}$ is given by

$$
\vec{f}=\vec{\xi} \times \vec{l}
$$

Thus the continuity of the $\xi$-vector at a two-dimensional corner represents a force balance. They used the notion of a force balance to analyse the more complicated situation of several interfaces intersecting at a point.

More recently Taylor (1992) stated an equivalent definition of the $\xi$-vector by employing a homogeneous extension of degree one of $\gamma(\vec{n})$ to arbitrary non-zero vectors $\vec{p}$,

$$
\gamma(\vec{p})=|\vec{p}| \gamma(\vec{p} /|\vec{p}|)
$$

where $\vec{n}$ denotes a unit normal, and showed that the $\xi$-vector may be defined by

$$
\xi^{j}=\frac{\partial \gamma(\vec{p})}{\partial p_{j}} .
$$

It follows from this definition that the $\xi$-vector is a homogeneous function of degree zero, and that

$$
\gamma(\vec{p})=\vec{p} \cdot \vec{\xi}(\vec{p})
$$

a property of the $\xi$-vector noted by Hoffman and Cahn for the case when $\vec{p}$ represents the unit normal-to the interface.

Wheeler and McFadden (1996) extended the $\xi$-vector theory to phase-field models with anisotropy introduced in the manner suggested by Caginalp and Fife (1986) and Kobayashi (1993). Both of these models employ free-energy functionals of the form (Taylor \& Cahn 1994)

$$
\mathcal{F}=\int\left\{\frac{1}{2}[\gamma(\nabla \phi)]^{2}+f(\phi, T)\right\} d V,
$$

where $f(\phi, T)$ is the bulk free energy per unit volume. The corresponding phase-field equation is given by the gradient flow

$$
\frac{1}{M} \frac{\partial \phi}{\partial t}=-\frac{\delta \mathcal{F}}{\delta \phi}=\nabla \cdot(\gamma \vec{\xi})-\frac{\partial f}{\partial \phi},
$$


where here and henceforth the argument of $\gamma$ is $\nabla \phi$ unless explicitly indicated otherwise. In contrast to the Cahn-Hoffmann theory for a sharp interface, the $\xi$-vector is now a vector field defined throughout the whole domain and not just on the interface. This $\xi$-vector formulation has been exploited to recover the appropriate three-dimensional form of the Gibbs-Thomson equation (7) in the sharp interface limit (Wheeler \& McFadden 1996).

\section{Generalised Stress Tensor}

The free energy functional (12) is a particular case of the more general expression

$$
\mathcal{I}=\int \mathcal{L}(\nabla \phi, \phi, \vec{x}) d V
$$

considered in variational treatments of continuous systems and fields (Goldstein 1980); $\mathcal{I}$ then represents the action and $\mathcal{L}$ represents the Lagrangian energy density, which is assumed to have explicit dependence on the scalar $\phi$, its gradient $\nabla \phi$, and the position vector $\vec{x}$. For an isothermal system with no applied fields, the integrand of the free energy functional (12) is independent of $\vec{x}$. In this case, energy-minimising equilibria admit an associated stress tensor and conservation law that result from the translational invariance of the Lagrangian; this is a simple consequence of Noether's theorem [see Goldstein (1980)].

Equilibrium states for $\mathcal{I}$ satisfy the Euler-Lagrange equation

$$
\frac{\partial \mathcal{L}}{\partial \phi}-\frac{\partial}{\partial x_{k}}\left[\frac{\partial \mathcal{L}}{\partial \phi_{, k}}\right]=0
$$

here we have employed the Einstein summation convention, and $\phi_{, k}$ denotes $\partial \phi / \partial x_{k}$.

A calculation gives

$$
\begin{gathered}
\frac{d \mathcal{L}}{d x_{j}}=\frac{\partial \mathcal{L}}{\partial \phi_{, k}} \phi_{, j k}+\frac{\partial \mathcal{L}}{\partial \phi} \phi_{, j}+\frac{\partial \mathcal{L}}{\partial x_{j}}=\frac{\partial \mathcal{L}}{\partial \phi_{, k}} \phi_{, j k}+\phi_{, j} \frac{d}{d x_{k}}\left[\frac{\partial \mathcal{L}}{\partial \phi_{, k}}\right]+\frac{\partial \mathcal{L}}{\partial x_{j}} \\
=\frac{d}{d x_{k}}\left[\phi_{, j} \frac{\partial \mathcal{L}}{\partial \phi_{, k}}\right]+\frac{\partial \mathcal{L}}{\partial x_{j}}
\end{gathered}
$$

so that

$$
-\frac{\partial \mathcal{L}}{\partial x_{j}}=\frac{d}{d x_{k}}\left[\phi_{, j} \frac{\partial \mathcal{L}}{\partial \phi_{, k}}-\delta_{j k} \mathcal{L}\right]
$$

where $\delta_{j k}$ is the Kronecker delta. Thus, if $\mathcal{L}$ has no explicit dependence on $x_{j}$, the stress tensor $\boldsymbol{\Xi}$, with components

$$
\Xi_{j k}=\phi_{, j} \frac{\partial \mathcal{L}}{\partial \phi_{, k}}-\delta_{j k} \mathcal{L}
$$


satisfies

$$
\nabla \cdot \Xi=0
$$

Since we have

$$
\mathcal{L}=\frac{1}{2} \gamma^{2}+f, \quad \frac{\partial \mathcal{L}}{\partial \phi_{, k}}=\gamma \xi_{k}
$$

this gives

$$
\Xi=\gamma \nabla \phi \otimes \vec{\xi}-\mathcal{L} \boldsymbol{I}
$$

where $\boldsymbol{I}$ is the unit tensor.

In general $\Xi$ is not symmetric, which generates an associated distributed body couple distribution given by $\gamma \vec{\xi} \times \nabla \phi$. This couple vanishes if the surface energy is isotropic (in which case $\vec{\xi}$ is parallel to $\nabla \phi$ ). Thus we see that the presence of surface energy anisotropy induces a body couple throughout the interface, indicating that the interface can reduce its surface energy by local rotation as well as by the mechanism of curve shortening which is only present in the isotropic case.

A simple calculation shows that $\nabla \phi$ is an eigenvector of $\Xi$ with eigenvalue $\left(\gamma^{2} / 2-f\right)$, and any vector $\vec{\xi}_{\perp}$ orthogonal to $\vec{\xi}$ is also an eigenvector with eigenvalue $-\left(\gamma^{2} / 2+f\right)$. Since $\nabla \phi \cdot \vec{\xi}=\gamma \neq 0$, these sets are linearly independent.

\subsection{The Sharp Interface Limit}

Here our aim is to determine the force per unit length acting on a line element in the interface in the sharp interface limit. It is well known that in this limit the leading order problem corresponds to that of a flat interface (Caginalp 1989). In view of this, and for the purposes of clarity we therefore conduct this analysis for the equivalent situation of the force acting on an infinite plane $\mathcal{A}$ with unit normal $\vec{a}$ intersecting a flat diffuse interface. For this leading order problem, $\phi$ only depends on $s$, which measures distance in the direction of the unit vector $\vec{n}$ that is normal to the contours of $\phi$, so that $\nabla \phi=\phi_{s} \vec{n}$ and $\gamma(\nabla \phi)=\phi_{s} \gamma(\vec{n})$. With our choice of scaling the surface energy is defined in terms of the integral of $\phi_{s}^{2}$, with

$$
\int_{-\infty}^{\infty} \phi_{s}^{2} d s=1 / \gamma(\vec{n})
$$


It is simple to show that the governing equation for $\phi$ has a first integral

$$
\frac{1}{2} \gamma(\vec{n})^{2} \phi_{s}^{2}-f(\phi, T)=0
$$

where $\phi_{s}$ denotes the derivative of $\phi$ with respect to $s$. It follows that the Lagrangian and stress tensor may be written as

$$
\mathcal{L}=\gamma(\vec{n})^{2} \phi_{s}^{2}, \quad \Xi=\phi_{s}^{2} \gamma(\vec{n})[\vec{n} \otimes \vec{\xi}-\gamma(\vec{n}) \boldsymbol{I}]
$$

The force per unit area, $\vec{F}$, acting on $\mathcal{A}$ is then given by

$$
\vec{F}=\Xi \cdot \vec{a}=\phi_{s}^{2} \gamma(\vec{n})[(\vec{\xi} \cdot \vec{a}) \vec{n}-\gamma(\vec{n}) \vec{a}]=\phi_{s}^{2} \gamma(\vec{n})[(\vec{\xi} \cdot \vec{a}) \vec{n}-(\vec{\xi} \cdot \vec{n}) \vec{a}]
$$

where we have employed (11). This may be written as

$$
\vec{F}=\phi_{s}^{2} \gamma(\vec{n}) \vec{\xi} \times \vec{l}
$$

where $\vec{l}=\vec{n} \times \vec{a}$.

We now take the surface $\mathcal{A}$ to be oriented perpendicular to the direction to the flat interface, so that the normal vector $\vec{a}$ is a tangent vector of the contours of $\phi$, and the intersection of $\mathcal{A}$ and the contours of $\phi$ lie in the direction of $\vec{l}$. To leading order, the total force per unit length, $\vec{f}$, in the direction of $\vec{l}$ is then obtained by integrating through the layer to give

$$
\vec{f}=\gamma(\vec{n})\left[\int_{-\infty}^{+\infty} \phi_{s}^{2} d s\right] \vec{\xi} \times \vec{l}
$$

or, using (22),

$$
\vec{f}=\vec{\xi} \times \vec{l}
$$

which reproduces the result given by Hoffman and Cahn for a sharp interface.

\section{Edges}

When the surface energy anisotropy is so pronounced that the $1 / \gamma$-plot is not convex, the Gibbs-Wulff construction gives that the equilibrium shapes of a sharp interface will include edges corresponding to missing orientations (see, e.g., Taylor 1992). In this section we consider the structure of edges in phase-field theory when the $1 / \gamma$-plot is not convex. 


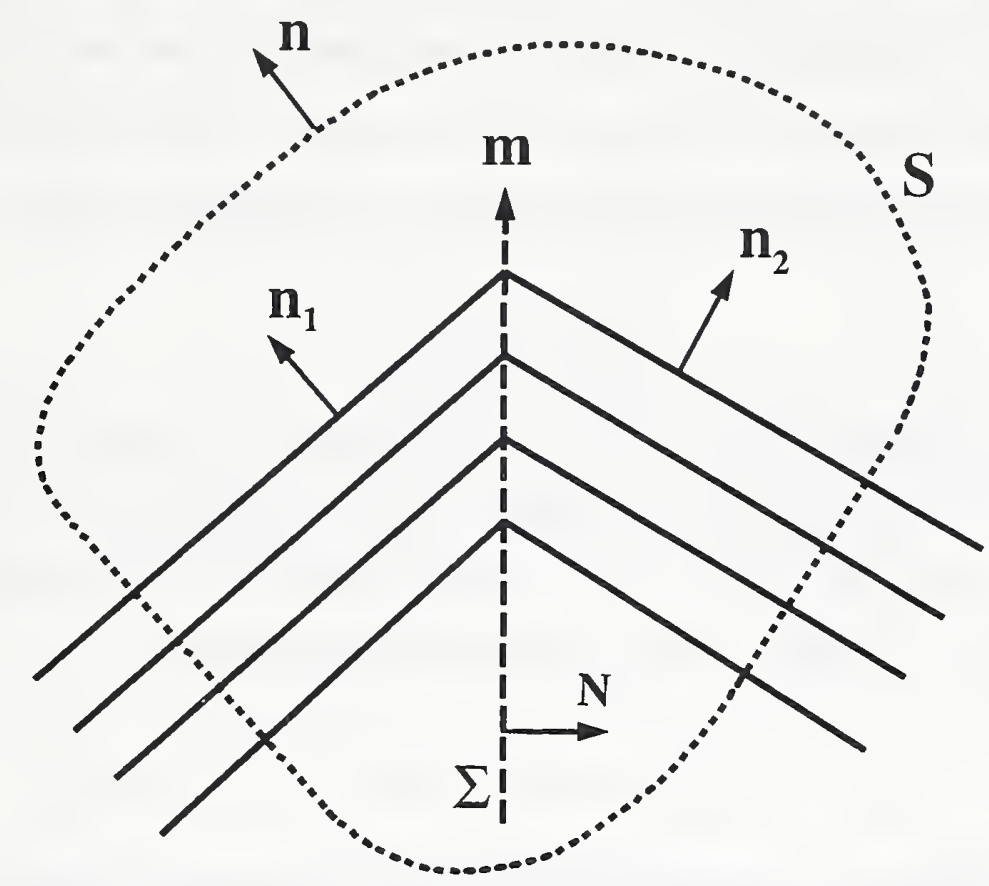

Figure 1: A schematic diagram of an edge. The control surface $S$ cuts the diffuse interfaces at right angles. The vector $\vec{l}$, in the direction of $\vec{n}_{1} \times \vec{n}_{2}$, points into the page. The vector $\vec{N}$ is the unit normal to the surface of discontinuity, $\Sigma$.

We consider the equilibrium situation where two stationary diffuse interfaces meet at an edge. As discussed further below, a basic assumption is that the phase field is everywhere continuous, although derivatives of $\phi$ are allowed to have surfaces of discontinuity representing a diffuse edge solution. In the next subsection we use the stress tensor to consider the far-field behaviour of the edge solution and, in particular, deduce the appropriate relation that determines the orientations of the two interfaces. In the subsequent subsection we investigate the solution structure in the region where the two interfaces meet.

\subsection{The Far-Field}

We denote the two interfaces as 1 and 2 and we assume that far away from the junction region they are planar with unit normals $\vec{n}_{1}$ and $\vec{n}_{2}$ as shown in Figure 1. Moreover we assume that the solution is translationally invariant in the direction of the unit vector $\vec{l}$ 
directed into the plane of the figure. We construct a cylindrical control surface $S$ which contains the junction region and intersects the two interfaces far away from the junction region; the contributions from the end caps of the cylinder can be disregarded. We assume that $S$ intersects the contours of $\phi$ at right angles. The divergence theorem yields

$$
\int_{S} \Xi \cdot \vec{n} d A=0
$$

where $\vec{n}$ is the outward normal to $S$. In the bulk phases away, from the interfacial regions, $\Xi$ is zero and so the only non-zero contributions are from the parts of $S$ that intersect the interfaces. We now take the sharp interface limit in which case the contribution from each interface is the same as considered above and so we conclude that

$$
\vec{\xi}_{1} \times \vec{l}+\vec{\xi}_{2} \times \vec{l}=0
$$

This is the form deduced by Cahn and Hoffman and corresponds to a force balance for the edge in the plane normal to $\vec{l}$.

\subsection{The Edge Structure}

When the $1 / \gamma$-plot is not convex the phase-field equation changes type from elliptic to hyperbolic [considered in two dimensions by Fife (1995), and in three dimensions by McFadden and Wheeler (1995)]. This suggests that the structure of the edge solutions may involve weak shocks. The variational principle for a diffuse interface can be used to deduce the jump conditions when edges are present; the conditions naturally arise when solutions with discontinuous derivatives are permitted.

We write the domain in the form $V=V_{1} \cup V_{2}$, where the phase-field variable $\phi$ is smooth in $V_{1}$ and $V_{2}$ and may have jumps across the common boundary, which we denote by the surface $\Sigma$; continuity of $\phi$ is imposed explicitly by using a Lagrange multiplier $\lambda$. The normal to $\Sigma$ is denoted by $\vec{N}$ and points into $V_{2}$. We minimise the free energy $\mathcal{F}$ subject to continuity of $\phi$ by writing

$$
0=\delta\left\{\int_{V_{1} \cup V_{2}}\left(\frac{1}{2}[\gamma(\nabla \phi)]^{2}+f\right) d V-\int_{\Sigma} \lambda\left[\phi_{1}-\phi_{2}\right] d A\right\}
$$

where the subscripts ' 1 ' and ' 2 ' denote evaluation of the function on either side of $\Sigma$. To 
obtain equilibrium shapes, a volume constraint should also be imposed, but this constraint does not effect the jump conditions which are our primary concern here.

Taking the variation gives

$$
\begin{gathered}
0=\int_{V_{1}}\left(\gamma \vec{\xi} \cdot \nabla \delta \phi+f_{\phi} \delta \phi\right) d V+\int_{V_{2}}\left(\gamma \vec{\xi} \cdot \nabla \delta \phi+f_{\phi} \delta \phi\right) d V+\int_{\Sigma}\left[\frac{1}{2} \gamma_{1}^{2}-\frac{1}{2} \gamma_{2}^{2}\right] \delta \nu d A \\
-\int_{\Sigma} \lambda\left[\delta \phi_{1}-\delta \phi_{2}\right] d A-\int_{\Sigma} \lambda\left\{\left[\frac{\partial \phi_{1}}{\partial N}-\frac{\partial \phi_{2}}{\partial N}\right]+\left[\phi_{1}-\phi_{2}\right] \mathcal{K}\right\} \delta \nu d A,
\end{gathered}
$$

where $\delta \nu$ is the outward variation of the surface $\Sigma$ along the normal direction, and $\mathcal{K}$ is the mean curvature of $\Sigma$. Integrating by parts the first two terms gives

$$
\begin{gathered}
0=\int_{V_{1}}\left(-\nabla \cdot[\gamma \vec{\xi}]+f_{\phi}\right) \delta \phi d V+\int_{V_{2}}\left(-\nabla \cdot[\gamma \vec{\xi}]+f_{\phi}\right) \delta \phi d V \\
+\int_{\Sigma}\left[\gamma_{1} \overrightarrow{\xi_{1}} \cdot \vec{N} \delta \phi_{1}-\gamma_{2} \overrightarrow{\xi_{2}} \cdot \vec{N} \delta \phi_{2}\right] d A+\int_{\Sigma}\left[\frac{1}{2} \gamma_{1}^{2}-\frac{1}{2} \gamma_{2}^{2}\right] \delta \nu d A \\
-\int_{\Sigma} \lambda\left[\delta \phi_{1}-\delta \phi_{2}\right] d A-\int_{\Sigma} \lambda\left\{\left[\frac{\partial \phi_{1}}{\partial N}-\frac{\partial \phi_{2}}{\partial N}\right]+\left[\phi_{1}-\phi_{2}\right] \mathcal{K}\right\} \delta \nu d A
\end{gathered}
$$

where we disregard any boundary terms other than those on $\Sigma$. The jump conditions across $\Sigma$ are therefore given by

$$
\begin{gathered}
\gamma_{1} \vec{\xi}_{1} \cdot \vec{N}=\lambda \\
\gamma_{2} \vec{\xi}_{2} \cdot \vec{N}=\lambda \\
{\left[\frac{1}{2} \gamma_{1}^{2}-\frac{1}{2} \gamma_{2}^{2}\right]=\lambda\left[\frac{\partial \phi_{1}}{\partial N}-\frac{\partial \phi_{2}}{\partial N}\right]+\lambda\left[\phi_{1}-\phi_{2}\right] \mathcal{K},}
\end{gathered}
$$

together with the continuity constraint

$$
\left[\phi_{1}-\phi_{2}\right]=0
$$

which removes the curvature dependence of the latter expression. Eliminating $\lambda$, the conditions resemble a common tangent condition (Cahn \& Carter 1996),

$$
\gamma_{1} \vec{\xi}_{1} \cdot \vec{N}=\gamma_{2} \vec{\xi}_{2} \cdot \vec{N}=\left[\frac{1}{2} \gamma_{1}^{2}-\frac{1}{2} \gamma_{2}^{2}\right] /\left[\frac{\partial \phi_{1}}{\partial N}-\frac{\partial \phi_{2}}{\partial N}\right]
$$

We emphasize that here $\gamma_{j}$ is short for $\gamma\left(\nabla \phi_{j}\right)$ as opposed to $\gamma\left(\overrightarrow{n_{j}}\right)$; since $\vec{\xi}$ is homogeneous of degree zero, this distinction is unnecessary for $\vec{\xi}$.

These jump conditions can be illustrated by the special case of a corner consisting of two flat diffuse interfaces with normals $\vec{n}_{1}$ and $\vec{n}_{2}$ joined together along a plane $\Sigma$, with 
normal vector $\vec{N}$, spanned by the orthogonal unit tangent vectors $\vec{m}$ and $\vec{l}$, where $\vec{l}$ lies in the direction of $\vec{n}_{1} \times \vec{n}_{2}$. Points in $\Sigma$ thus have the form $\vec{R}_{0}+s_{1} \vec{m}+s_{2} \vec{l}$, where $\vec{R}_{0}$ is a fixed point in $\Sigma$. It is convenient to write the one-dimensional solution $\phi(s)$ for a flat interface in the form $\phi(s)=g(s / \gamma(\vec{n}))$; [note that the scaling for the argument of $g$ is apparent from Eq. (23)]. Hence the solutions on either side of $\Sigma$ can be written in the form

$$
\phi_{1}(\vec{x})=g\left(\frac{\left[\vec{x}-\vec{R}_{0}\right] \cdot \vec{n}_{1}}{\gamma\left(\vec{n}_{1}\right)}\right), \quad \phi_{2}(\vec{x})=g\left(\frac{\left[\vec{x}-\vec{R}_{0}\right] \cdot \vec{n}_{2}}{\gamma\left(\vec{n}_{2}\right)}\right)
$$

Setting $\phi_{1}=\phi_{2}$ at a point $\vec{x}=\vec{R}_{0}+s_{1} \vec{m}+s_{2} \vec{l}$ in $\Sigma$ and using $\vec{n}_{1} \cdot \vec{l}=\vec{n}_{2} \cdot \vec{l}=0$ gives

$$
\frac{\vec{m} \cdot \vec{n}_{1}}{\gamma\left(\vec{n}_{1}\right)}=\frac{\vec{m} \cdot \vec{n}_{2}}{\gamma\left(\vec{n}_{2}\right)}
$$

We have $\left|\nabla \phi_{1}\right|=g^{\prime} / \gamma\left(\vec{n}_{1}\right)$, etc., so that from homogeneity we obtain $\gamma\left(\nabla \phi_{1}\right)=\left|\nabla \phi_{1}\right| \gamma\left(\vec{n}_{1}\right)=$ $g^{\prime}=\gamma\left(\nabla \phi_{2}\right)$; i.e., $\gamma(\nabla \phi)$ is continuous across $\Sigma$. The jump conditions (38) then imply

$$
\vec{\xi}_{1} \cdot \vec{N}=0, \quad \vec{\xi}_{2} \cdot \vec{N}=0
$$

Thus $\vec{\xi}_{1}$ and $\vec{\xi}_{2}$ both lie in the plane $\Sigma$, and we write

$$
\vec{\xi}_{1}=\alpha_{1} \vec{m}+\beta_{1} \vec{l}, \quad \vec{\xi}_{2}=\alpha_{2} \vec{m}+\beta_{2} \vec{l}
$$

for some $\alpha_{1}, \alpha_{2}, \beta_{1}$ and $\beta_{2}$. We then note that

$$
\gamma\left(\vec{n}_{1}\right)=\vec{\xi}_{1} \cdot \vec{n}_{1}=\alpha_{1} \vec{m} \cdot \vec{n}_{1}, \quad \gamma\left(\vec{n}_{2}\right)=\vec{\xi}_{2} \cdot \vec{n}_{2}=\alpha_{2} \vec{m} \cdot \vec{n}_{2}
$$

so that by using Eq. (40) we find $\alpha_{1}=\alpha_{2}$. Hence we deduce that

$$
\left(\vec{\xi}_{1}-\vec{\xi}_{2}\right) \times \vec{l}=0
$$

which is the form stated by Hoffman and Cahn. It follows that $\vec{m}$ is simply given by

$$
\vec{m}=\vec{\xi}_{1}-\left[\vec{\xi}_{1} \cdot \vec{l}\right] \vec{l}=\vec{\xi}_{2}-\left[\vec{\xi}_{2} \cdot \vec{l}\right] \vec{l}
$$

i.e., the plane $\Sigma$ is contained by $\vec{l}$ and the common component of the two $\xi$-vectors which is orthogonal to $\vec{l}$. 


\section{4. $\vec{\xi}$-vector Formulation of Multiple Order Parameter Models}

We next consider a multiple order parameter description of an isothermal phase transition which involves $N$ order parameters, $X_{1}, X_{2}, \ldots, X_{N}$. We assume that the underlying freeenergy functional can be expressed in the form

$$
\mathcal{F}=\int_{V}\left\{\frac{1}{2} \sum_{i=1}^{i=N}\left[\gamma_{i}\left(\nabla X_{i}\right)\right]^{2}+f(\mathbf{X}, T)\right\} d V
$$

where $f(\mathbf{X}, T)$ is the bulk free-energy density, $\mathbf{X}$ represents the $N$-tuple $\left(X_{1}, X_{2}, \ldots, X_{N}\right)$, $T$ is temperature and the functions $\gamma_{i}\left(\nabla X_{i}\right)$ represent the gradient energy contributions to the free energy of the system associated with each order parameter $X_{i}$ and are assumed to be homogeneous functions of degree one.

We further assume that the order parameters are nonconserved quantities and hence that the dynamics of the system may be given by the gradient flow

$$
\frac{\partial X_{i}}{\partial t}=-M_{i} \frac{\delta \mathcal{F}}{\delta X_{i}}
$$

for $i=1, \ldots N$, where the $M_{i}$ are positive constants. Evaluating the variational derivatives gives that the $N$ governing equations may be written as

$$
\frac{1}{M_{i}} \frac{\partial X_{i}}{\partial t}=\nabla \cdot\left[\gamma_{i} \vec{\xi}_{i}\right]-\frac{\partial f}{\partial X_{i}}
$$

where $\vec{\xi}_{i}$ will be called the 'sub- $\xi$-vector' associated with the order parameter $X_{i}$ whose contravariant components are defined by the relation

$$
\xi_{i}^{j}=\frac{\partial \gamma_{i}}{\partial X_{i, j}}
$$

it then follows from homogeneity that each sub- $\xi$-vector satisfies $\vec{\xi}_{i} \cdot \vec{p}=\gamma_{i}(\vec{p})$. Here $X_{i, j}$ denotes the partial derivative of $X_{i}$ with respect to the cartesian coordinate $x_{j}$. The function $f(\mathrm{X}, T)$ has multiple-well structure, with each corresponding minimum representing a stable bulk phase; the gradient energy terms allow solutions wherein distinct bulk phases are separated by a diffuse interface. We limit our attention to situations where the solution is unique for a given orientation of the interface, and further assume that the interface has no transverse structure. The governing equations are a system of phase-field equations that are 
coupled through the bulk free energy term. The class of diffuse interface theories which may be represented by the free-energy functional given by Eq. (46) include phase-field models as well as multiple-order parameter models such as the fcc model discussed in the Introduction (Braun et al. 1996). In this case an appropriate expression for the functions $\gamma_{i}$, which are homogeneous of degree one, is the quadratic form

$$
\gamma_{i}=\left\{\left[\nabla X_{i}\right]^{T} \cdot A_{i} \cdot \nabla X_{i}\right\}^{1 / 2}
$$

where $A_{i}$ are positive definite matrices with constant coefficients. For the fcc model of Braun et al. we have three order parameters; $N=3$, and

$$
A_{1}=\left(\begin{array}{ccc}
A & 0 & 0 \\
0 & B & 0 \\
0 & 0 & B
\end{array}\right), \quad A_{2}=\left(\begin{array}{ccc}
B & 0 & 0 \\
0 & A & 0 \\
0 & 0 & B
\end{array}\right), \quad A_{3}=\left(\begin{array}{ccc}
B & 0 & 0 \\
0 & B & 0 \\
0 & 0 & A
\end{array}\right),
$$

where $A$ and $B$ are positive constants.

\subsection{The Sharp Interface Limit}

Here we consider the situation where a thin, curved, moving interface separates two bulk states, denoted by $\mathbf{X}=\mathbf{X}_{+\infty}$ and $\mathbf{X}=\mathbf{X}_{-\infty}$, where $f_{, X_{i}}\left(\mathbf{X}_{+\infty}, T_{c}\right)=f_{, X_{i}}\left(\mathbf{X}_{-\infty}, T_{c}\right)=0$, and $T_{c}$ is the temperature at which the phase transition occurs, so that $f\left(\mathbf{X}_{+\infty}, T_{c}\right)=$ $f\left(\mathbf{X}_{-\infty}, T_{c}\right)=0$ as well. We consider the temperature to be close to $T_{c}$, so that to a good approximation we may assume that the bulk free-energy density is linearly dependent on the temperature. We non-dimensionalise so that the phase transition occurs at a value of the dimensionless temperature of unity, and represent the dimensionless free energy as

$$
\mathcal{F}=\int_{V}\left\{\frac{\epsilon}{2} \sum_{i=1}^{i=N}\left[\Gamma_{i}\left(\nabla X_{i}\right)\right]^{2}+f(\mathbf{X}, T)\right\} d V
$$

where

$$
f(\mathbf{X}, T)=\frac{1}{\epsilon} f^{(-1)}(\mathbf{X})-(T-1) s^{(0)}(\mathbf{X}) .
$$

Here $\epsilon$ characterises the thickness of the interface, $\Gamma_{i}\left(\nabla X_{i}\right)$ is the dimensionless form of $\gamma_{i}, s^{(0)}$ is an entropy density, and all quantities here and henceforth in this subsection are dimensionless. 
The dimensionless governing equations are then

$$
\frac{1}{M_{i}} \frac{\partial X_{i}}{\partial t}=\epsilon \nabla \cdot\left[\Gamma_{i}\left(\nabla X_{i}\right) \vec{\xi}_{i}\right]-f_{, X_{i}}
$$

We introduce a surface-fitted curvilinear coordinate system $\left\{u^{1}, u^{2}, u^{3}\right\}$ located within the interface (see, e.g., Wheeler \& McFadden 1996). To this end we may, without loss of generality, define a surface within the interface, denoted by $S$, by the level set $X_{1}\left(x^{1}, x^{2}, x^{3}\right)=$ constant (the average of its bulk values, say) given by

$$
\vec{x}=\vec{R}\left(u^{1}, u^{2}\right)+u^{3} \vec{n}\left(u^{1}, u^{2}\right),
$$

where $\vec{x}=\vec{R}\left(u^{1}, u^{2}\right)$ represents $S, u^{1}$ and $u^{2}$ are surface coordinates of $S$, and $\vec{n}\left(u^{1}, u^{2}\right)$ is its unit normal.

To proceed with our asymptotic analysis in the limit $\epsilon \rightarrow 0$, we rescale distance through the interface with respect to the interfacial thickness and so put $u^{3}=\epsilon \rho$. We expand the field variables as regular perturbation series in $\epsilon$

$$
\begin{gathered}
X_{i}=X_{i}^{(0)}\left(u^{1}, u^{2}, \rho\right)+\epsilon X_{i}^{(1)}\left(u^{1}, u^{2}, \rho\right)+\mathcal{O}\left(\epsilon^{2}\right), \\
T=1+\epsilon T^{(1)}+\mathcal{O}\left(\epsilon^{2}\right) .
\end{gathered}
$$

The asymptotic representation of $\Gamma_{i}\left(\nabla X_{i}\right)$ and the sub- $\xi$-vector in the layer are given by

$$
\begin{aligned}
& \Gamma_{i}\left(\nabla X_{i}\right)=\frac{1}{\epsilon} \Gamma_{i}(\vec{n}) X_{i, \rho}^{(0)}+\Gamma_{i}(\vec{n}) X_{i, \rho}^{(1)}+\vec{\xi}_{i}(\vec{n}) \cdot \nabla_{S} X_{i}^{(0)}+\mathcal{O}(\epsilon), \\
& \xi_{i}^{3}=\Gamma_{i}(\vec{n})+\mathcal{O}\left(\epsilon^{2}\right)
\end{aligned}
$$

[see Wheeler and McFadden (1996), where they were derived for the case of a scalar anisotropic phase-field model.]

\subsubsection{The Leading Order Problem}

The leading order problem is

$$
\left[\Gamma_{i}(\vec{n})\right]^{2} \frac{\partial^{2} X_{i}^{(0)}}{\partial \rho^{2}}-\frac{\partial f^{(-1)}\left(\mathbf{X}^{(0)}\right)}{\partial X_{i}}=0
$$

with the far field boundary conditions $\mathrm{X}^{(0)} \rightarrow \mathrm{X}_{ \pm \infty}$ as $\rho \rightarrow \pm \infty$; we write the solution in the form $X_{i}^{(0)}=X_{i}^{(0)}(\rho ; \vec{n})$ to emphasize the dependence of the solution on the unit normal vector $\vec{n}$. 
We note there is a first integral

$$
\frac{1}{2} \sum_{i=1}^{N}\left[\Gamma_{i}(\vec{n})\right]^{2}\left[X_{i, \rho}^{(0)}\right]^{2}=f^{(-1)}\left(\mathbf{X}^{(0)}\right)
$$

The surface energy, $\gamma(\vec{n})$, which depends on the orientation of the surface, is given by

$$
\gamma(\vec{n})=\int_{-\infty}^{+\infty}\left\{\frac{1}{2} \sum_{i=1}^{N}\left[\Gamma_{i}(\vec{n})\right]^{2}\left[X_{i, \rho}^{(0)}\right]^{2}+f^{(-1)}\left(\mathbf{X}^{(0)}\right)\right\} d \rho
$$

which, on using the first integral, may be written as

$$
\gamma(\vec{n})=\sum_{i=1}^{N}\left[\Gamma_{i}(\vec{n})\right]^{2} \mathcal{I}_{i}(\vec{n})
$$

where we define

$$
\mathcal{I}_{i}(\vec{n})=\int_{-\infty}^{+\infty}\left[X_{i, \rho}^{(0)}(\rho ; \vec{n})\right]^{2} d \rho .
$$

Unlike the scalar case, the surface energy cannot be determined explicitly, but requires knowledge of the leading order solution to evaluate the integrals $\mathcal{I}_{i}(\vec{n})$ (see also Barroso $\&$ Fonseca 1994). However, we next show that we may nevertheless determine a formal expression for the appropriate $\xi$-vector.

To this end we first make a homogeneous first order extension of the surface energy. This may be done in a natural manner by extending the leading order solution to Eq. (60), in the following way:

For an arbitrary non-zero vector $\vec{p}$, let $X_{i}^{(0)}(\rho ; \vec{p})=X_{i}^{(0)}(\rho /|\vec{p}| ; \vec{p} /|\vec{p}|)$; by using the homogeneity of $\Gamma_{i}(\vec{p})$, the function $X_{i}^{(0)}(\rho ; \vec{p})$ then satisfies the equation

$$
\left[\Gamma_{i}(\vec{p})\right]^{2} \frac{\partial^{2} X_{i}^{(0)}}{\partial \rho^{2}}-\frac{\partial f^{(-1)}\left(\mathbf{X}^{(0)}\right)}{\partial X_{i}}=0, \text { for } i=1, \ldots, N,
$$

with the same far-field boundary conditions as the original leading-order problem.

We now extend the definition of the surface energy given by Eq. (62) as

$$
\gamma(\vec{p})=\sum_{i=1}^{N}\left[\Gamma_{i}(\vec{p})\right]^{2} \mathcal{I}_{i}(\vec{p})
$$

where we define

$$
\mathcal{I}_{i}(\vec{p})=\int_{-\infty}^{+\infty}\left[X_{i, \rho}^{(0)}(\rho ; \vec{p})\right]^{2} d \rho
$$


It is straightforward to show that this definition is the homogeneous extension of order one of the surface energy to non-unit vectors. Using Eq. (61) it may also be written as

$$
\gamma(\vec{p})=2 \int_{-\infty}^{+\infty} f^{(-1)}\left(\mathrm{X}^{(0)}(\rho ; \vec{p})\right) d \rho .
$$

Employing the definition of the $\xi$-vector, Eq. (10), and the form for the surface energy given by Eq. (68) we find

$$
\xi^{j}=\frac{\partial \gamma(\vec{p})}{\partial p_{j}}=2 \int_{-\infty}^{+\infty} \sum_{i=1}^{N} \frac{\partial f^{(-1)}}{\partial X_{i}^{(0)}} \frac{\partial X_{i}^{(0)}}{\partial p_{j}} d \rho
$$

Taking the scalar product of the governing equations $(65)$ for $X_{i}^{(0)}(\rho ; \vec{p})$ with $X_{i, p_{j}}^{(0)}$ gives that

$$
\sum_{i=1}^{N}\left[\Gamma_{i}(\vec{p})\right]^{2} \frac{\partial^{2} X_{i}^{(0)}}{\partial \rho^{2}} \frac{\partial X_{i}^{(0)}}{\partial p_{j}}=\sum_{i=1}^{N} \frac{\partial f^{(-1)}}{\partial X_{i}^{(0)}} \frac{\partial X_{i}^{(0)}}{\partial p_{j}},
$$

in which case we find that

$$
\xi^{j}=2 \sum_{i=1}^{N}\left[\Gamma_{i}(\vec{p})\right]^{2} \int_{-\infty}^{+\infty} \frac{\partial^{2} X_{i}^{(0)}}{\partial \rho^{2}} \frac{\partial X_{i}^{(0)}}{\partial p_{j}} d \rho
$$

Integrating by parts then yields that

$$
\xi^{j}=-2 \sum_{i=1}^{N}\left[\Gamma_{i}(\vec{p})\right]^{2} \int_{-\infty}^{+\infty} \frac{\partial X_{i}^{(0)}}{\partial \rho} \frac{\partial^{2} X_{i}^{(0)}}{\partial \rho \partial p_{j}} d \rho
$$

which may be expressed as

$$
\xi^{j}=-\sum_{i=1}^{N}\left[\Gamma_{i}(\vec{p})\right]^{2} \frac{\partial \mathcal{I}_{i}(\vec{p})}{\partial p_{j}} .
$$

Using the alternative representation of the surface energy given by Eq. (66) in the definition of the $\xi$-vector, Eq. (10), we find

$$
\xi^{j}=\frac{\partial \gamma}{\partial p_{j}}=2 \sum_{i=1}^{N} \xi_{i}^{j} \Gamma_{i}(\vec{p}) \mathcal{I}_{i}(\vec{p})+\sum_{i=1}^{N}\left[\Gamma_{i}(\vec{p})\right]^{2} \frac{\partial \mathcal{I}_{i}(\vec{p})}{\partial p_{j}} .
$$

Using Eq. (73) and rearranging we find that

$$
\vec{\xi}=\sum_{i=1}^{N} \vec{\xi}_{i} \Gamma_{i}(\vec{p}) \mathcal{I}_{i}(\vec{p}) .
$$

We observe that the $\xi$-vector is linear combination of the sub- $\xi$-vectors. Using Eq. (66) and the homogeneity of $\Gamma_{i}(\vec{p})$, we also have that the $\xi$-vector satisfies $\vec{\xi} \cdot \vec{p}=\gamma(\vec{p})$. We next show that this definition of the $\xi$-vector is consistent with the Gibbs-Thomson equation that is recovered in the sharp-interface limit. 


\subsubsection{The First Order Problem}

The first order problem is (see Wheeler and McFadden 1996)

$$
\begin{aligned}
& {\left[\Gamma_{i}(\vec{n})\right]^{2} \frac{\partial^{2} X_{i}^{(1)}}{\partial \rho^{2}}-\sum_{k=1}^{N} \frac{\partial^{2} f^{(-1)}\left(\mathbf{X}^{(0)}\right)}{\partial X_{i} X_{k}} X_{k}^{(1)}=-T^{(1)} \frac{\partial s^{(0)}\left(\mathrm{X}^{(0)}\right)}{\partial X_{i}} } \\
& \quad-\frac{1}{M_{i}} v_{n} X_{i, \rho}^{(0)}-\Gamma_{i}(\vec{n}) \vec{\xi}_{i}(\vec{n}) \cdot \nabla_{S} X_{i, \rho}^{(0)}-\nabla_{S} \cdot\left[\Gamma_{i}(\vec{n}) \vec{\xi}_{i}(\vec{n}) X_{i, \rho}^{(0)}\right],
\end{aligned}
$$

where $v_{n}$ is the nroaml velocity of $S$. The left-hand side is self-adjoint with homogeneous solution given by $\left(X_{1, \rho}^{(0)}, X_{2, \rho}^{(0)}, \ldots, X_{N, \rho}^{(0)}\right)$. The solvability condition, formed from the inner product of the adjoint solution with the right-hand side, is

$$
\begin{aligned}
-v_{n} \sum_{i=1}^{N} \frac{1}{M_{i}} \int_{-\infty}^{+\infty}\left[X_{i, \rho}^{(0)}\right]^{2} d \rho=T^{(1)} \Delta s & +\sum_{i=1}^{N} \Gamma_{i}(\vec{n}) \vec{\xi}_{i}(\vec{n}) \cdot \int_{-\infty}^{+\infty} X_{i, \rho}^{(0)} \nabla_{S} X_{i, \rho}^{(0)} d \rho \\
& +\sum_{i=1}^{N} \int_{-\infty}^{+\infty} X_{i, \rho}^{(0)} \nabla_{S} \cdot\left[\Gamma_{i}(\vec{n}) \vec{\xi}_{i}(\vec{n}) X_{i, \rho}^{(0)}\right] d \rho
\end{aligned}
$$

where $\Delta s=s^{(0)}\left(\mathrm{X}_{+\infty}\right)-s^{(0)}\left(\mathrm{X}_{-\infty}\right)$ is the entropy density difference between the two bulk phases. We now consider in detail the latter two terms on the right-hand side that involve $\nabla_{S}$. They may be written as

$$
\sum_{i=1}^{N}\left[\frac{1}{2} \Gamma_{i}(\vec{n}) \vec{\xi}_{i}(\vec{n}) \cdot \nabla_{S} \mathcal{I}_{i}+\mathcal{I}_{i} \nabla_{S} \cdot\left[\Gamma_{i}(\vec{n}) \vec{\xi}_{i}(\vec{n})\right]+\int_{-\infty}^{+\infty} X_{i, \rho}^{(0)} \Gamma_{i}(\vec{n}) \vec{\xi}_{i}(\vec{n}) \cdot \nabla_{S} X_{i, \rho}^{(0)} d \rho\right]
$$

where we have expanded the $\nabla_{S}$ operator in the second term of the right-hand side of Eq. (77) to obtain the last two terms in the above expression. The above expression may be written as

$$
-\sum_{i=1}^{N}\left[\frac{1}{2} \Gamma_{i}(\vec{n}) \vec{\xi}_{i}(\vec{n}) \cdot \nabla_{S} \mathcal{I}_{i}+\mathcal{I}_{i} \nabla_{S} \cdot\left[\Gamma_{i}(\vec{n}) \vec{\xi}_{i}(\vec{n})\right]+\frac{1}{2} \Gamma_{i}(\vec{n}) \vec{\xi}_{i}(\vec{n}) \cdot \nabla_{S} \mathcal{I}_{i}\right]
$$

Noting the first and last terms are identical this simplifies to

$$
\sum_{i=1}^{N} \nabla_{S} \cdot\left[\mathcal{I}_{i}(\vec{n}) \Gamma_{i}(\vec{n}) \vec{\xi}_{i}(\vec{n})\right]
$$

i.e., $\nabla_{S} \cdot \vec{\xi}(\vec{n})$ on using Eq. (75). Hence the solvability condition, Eq. (77), may be written as

$$
T^{(1)}=-\frac{1}{\Delta s} \nabla_{S} \cdot \vec{\xi}(\vec{n})-\frac{1}{\mu(\vec{n})} v_{n}
$$

where

$$
\frac{1}{\mu(\vec{n})}=\frac{1}{\Delta s} \sum_{i=1}^{N} \frac{\mathcal{I}_{i}(\vec{n})}{M_{i}} .
$$




\subsection{Generalised Stress Tensor}

We next consider the form of the generalised stress tensor for the multiple-order parameter case. The appropriate dimensional Lagrangian density has the form

$$
\mathcal{L}(\mathbf{X}, \nabla \mathbf{X})=\frac{1}{2} \sum_{i=1}^{i=N}\left[\gamma_{i}\left(\nabla X_{i}\right)\right]^{2}+f(\mathbf{X}, T)
$$

which is again translationally invariant. A calculation analogous to that done above for the scalar case shows that the corresponding stress tensor has the form

$$
\Xi=\sum_{i=1}^{N} \gamma_{i} \nabla X_{i} \otimes \vec{\xi}_{i}-\mathcal{L} \boldsymbol{I}
$$

and satisfies $\nabla \cdot \boldsymbol{\Xi}=0$.

Considering as before the force acting on an infinite plane $\mathcal{A}$, with unit normal $\vec{a}$, intersecting at right angles a flat diffuse interface with normal vector $\vec{n}$, a straight-forward calculation gives that the integrated force per unit length $\vec{f}$ in the direction $\vec{l}=\vec{n} \times \vec{a}$ is given by the analogous expression

$$
\vec{f}=\vec{\xi} \times \vec{l}
$$

in the multiple-order-parameter case as well.

We now consider a diffuse stationary $n$-junction which corresponds in the sharp interface limit to a straight line (denoted by $\vec{l}$, say) at which $n$ stable bulk phases and their $n$ planar interfaces meet. Let the $n$ bulk phases be denoted by $\mathbf{X}_{I}, \mathbf{X}_{I I}$, etc., indexed in an anticlockwise fashion about the junction and let the $n \xi$-vectors associated with each diffuse interface be denoted by $\vec{\xi}_{I}, \vec{\xi}_{I I}$, etc., where $\vec{\xi}_{I}$ denotes the $\xi$-vector between the bulk phases $\mathbf{X}_{I}$ and $\mathbf{X}_{I I}$, etc. Then a calculation similar to that given above for the scalar case, based on the divergence theorem for a suitable control volume, gives the result

$$
\vec{l} \times\left(\vec{\xi}_{I}+\vec{\xi}_{I I}+\ldots\right)=0
$$

This is the same result as given by Hoffman and Cahn. It determines the equilibrium angles of the $n$-junction and represents a force balance. 


\section{Discussion}

For the phase-field model $(N=1)$ the surface energy is directly related to the gradient energy coefficient, so that a desired anisotropic surface energy can be obtained a priori by selecting the gradient energy appropriately. For the general case $(N>1)$ involving multiple order parameters this is no longer true; the surface energy is proportional to a weighted sum of the gradient energy coefficients, with weights that depend on the specific form taken by the solution of the leading order problem Eq. (60). In this approach the surface energy is inherited from the model, which may reflect the underlying crystallographic symmetries of the atomic lattice of the material in question, as in the case of the fcc model developed by Braun et al. (1996). In contrast to the phase-field model in which the surface energy anisotropy is specified a priori, multiple order parameter models seek to provide a more detailed description of the structure of the interface and as a result predict the surface energy anisotropy.

This difference of methodology between phase-field models and multiple order parameter models is also reflected in the $\xi$-vector. For the phase-field case $(N=1)$, in the same way that the surface energy anisotropy is specified a priori, so is the $\xi$-vector. In contrast, in the case of multiple order parameter models $(N>1)$ this is not so. Although for each order parameter a sub- $\xi$-vector may be defined which is a vector field independent of the value of the $\epsilon$, the $\xi$-vector for the whole system only emerges in the sharp interface limit as a linear combination of the sub- $\xi$-vectors whose coefficients depend on the functionals $\mathcal{I}_{i}$ and so, like the surface energy, requires the leading order contributions to the order parameters. In contrast the stress tensor is defined independently of taking the sharp interface limit.

We observe that, although we have taken the dynamics of the system to be a simple gradient flow with rate constants $M_{i}$ that are independent of the orientation of the level sets of the order parameters, the dynamics of the interface in the sharp interface limit involves a mobility $\mu(\vec{n})$ which depends on the orientation of the interface. Comparing the expression for $\mu(\vec{n})$, Eq. (82), with that for $\gamma(\vec{n})$, Eq. (63), we note that the form of the kinetic anisotropy generally differs from that of the surface energy anisotropy.

We may also determine the form of the stress tensor associated with a conserved order 
parameter, such as the concentration, which for example occurs in the Cahn-Hilliard theory of spinodal decomposition (Cahn \& Hilliard 1958). In this case the associated stress tensor is

$$
\mathbf{T}_{C}=\delta^{2} \nabla c \otimes \nabla c-\left\{\delta^{2}\left[c \nabla^{2} c+\frac{1}{2}|\nabla c|^{2}\right]+f(c)-c f_{c}(c)\right\} \boldsymbol{I},
$$

where, $c$ is the concentration, $f(c)$ is the bulk free-energy density and $\delta^{2} / 2$ is the gradient energy coefficient.

For the case of isotropic surface energy the stress tensor $\boldsymbol{\Xi}$ for the phase-field model has the form $\gamma \nabla \phi \otimes \nabla \phi-\mathcal{L} \boldsymbol{I}$. Both this tensor and the stress tensor for a conserved order parameter are similar to the Korteweg stress tensor $\mathbf{T}_{K}$ (see. e.g., Truesdell and Noll 1965) that occurs in the theory of fluids near close to their critical point (Anderson and McFadden 1996),

$$
\mathrm{T}_{K}=-\nabla \rho \otimes \nabla \rho+\left(\rho \nabla^{2} \rho+\frac{1}{2}|\nabla \rho|^{2}\right) \boldsymbol{I}
$$

where in this situation the density of the fluid, $\rho$, provides the natural order parameter. They are also similar in form to the Maxwell stress tensor $\mathbf{T}_{M}$ associated with a electromagnetic field, which has the form (Jackson 1975)

$$
\mathbf{T}_{M}=\frac{1}{4 \pi}\left(\vec{E} \otimes \vec{E}+\vec{B} \otimes \vec{B}-\frac{1}{2}\left[E^{2}+B^{2}\right] \boldsymbol{I}\right),
$$

where $\vec{E}$ and $\vec{B}$ are the electric field and magnetic induction, respectively, and $\left[E^{2}+B^{2}\right] / 2$ represents the energy density. The tensors $\mathbf{T}_{C}, \mathrm{~T}_{K}$, and $\mathbf{T}_{M}$ are all symmetric, which is a consequence of the isotropic form of the associated Lagrangians; in contrast, $\boldsymbol{\Xi}$ is generally nonsymmetric, which results from the anisotropic nature of the free energy functional.

In general it may be shown that the phase-field stress tensor derived above does not contribute to the local entropy production. A more complete theory would incorporate deformation of matter which would in general provide additional dissipative contributions to the stress tensor (such as a viscous stress), and would also provide a possible means of balancing the distributed body couple associated with anisotropic surface energy.

The recovery of the equilibrium configuration of line junctions in the sharp interface limit of diffuse interface models has previously been limited to situations in which the surface energy is isotropic (see, e.g., Owen et al. 1990, Bronsard \& Reitich 1993, Wheeler et al. 1996, and Novick-Cohen \& Cahn 1996). These treatments employed identities that are equivalent 
to the conservation law, $\nabla \cdot \boldsymbol{\Xi}=0$, but without explicitly identifying the stress tensor $\boldsymbol{\Xi}$ or the physical basis of the conservation law, as we have done in this paper for the more general anisotropic case.

\section{Conclusion}

In conclusion, the approach adopted demonstrates that both multiple order parameter models and less physically-based phase-field models share a common framework, the former providing a generalisation of the notion of the $\xi$-vector for phase-field models as well as providing a connection to the classical $\xi$-vector theory for a sharp interface. Moreover, we have identified an associated stress tensor, $\boldsymbol{\Xi}$, which gives the stress throughout the interfacial layer and may be used to make a connection, in the sharp interface limit, with the forces acting in the interface, through the notion of the $\xi$-vector. It enables us to derive the equilibrium conditions for edges and $n$-junctions in terms of a force balance. Lastly, we have shown that edge formation in a phase-field model is characterised by the formation of a weak shock across which the components of the $\xi$-vector normal to the direction of the edge are continuous, which again may be interpreted as a force balance. In all of the aspects of the interface that we have considered, the notion of the $\xi$-vector and the stress tensor play a central rôle.

\section{Acknowledgements}

The authors are grateful for useful discussions with D. M. Anderson, J. W. Cahn, S. R. Coriell, R. E. Craine, P. C. Fife, R. F. Sekerka and J. E. Taylor. This work was partially supported by the Applied and Computational Mathematics Program of ARPA, by the Microgravity Science and Applications Program of NASA, and by a NATO collaborative research grant.

\section{References}

[1] Allen, S. M., and Cahn, J. W. 1979 A microscopic theory for antiphase boundary motion and its application to antiphase domain coarsening. Acta metall. mater. 27, pp. 10851095. 
[2] Anderson, D. M., and McFadden, G.B. 1996 Preprint.

[3] Barroso, Ana Cristina, and Fonseca, Irene 1994 Anisotropic singular perturbations the vectorial case. Proc. Roy. Soc. Edin. 124A, pp. 527-571.

[4] Braun, R. J., Cahn, J. W., McFadden, G. B., and Wheeler, A. A. 1996 Anisotropy of interfaces in an ordered alloy: A multiple-order-parameter model. Submitted to the Phil. Trans. Roy. Soc. Lond..

[5] Braun, R. J., Cahn, J. W., McFadden, G. B., Rushmeier, H. E., and Wheeler, A. A., 1996 Anisotropic equilibrium and growth shapes in the ordering of an fcc alloy. (In preparation).

[6] Bronsard, Lia and Reitich, Fernando 1993 On three-phase boundary motion and the singular limit of a vector-valued Ginzburg-Landau equation. Arch. Rational Mech. Anal. 124 pp. 355-379.

[7] Caginalp, G. 1989 Stefan and Hele-Shaw type models as asymptotic limits of the phasefield equations. Phys. Rev. A 39, pp 5887-5896.

[8] Caginalp, Gunduz and Fife, Paul 1986 Phase-field methods for interfacial boundaries. Phys. Rev. B 33, pp. 7792-7794.

[9] Cahn, J. W. and Allen, S. M. 1977 A microscopic theory for domain wall motion and its experimental verification in $\mathrm{Fe}-\mathrm{Al}$ alloy domain growth kinetics. J. Phys. (Paris) Colloque C7, pp. C7-51-C7-54.

[10] Cahn, J. W., and Carter, W. C. 1996 Crystal Shapes and Phase Equilibria: A Common Mathematical Basis Met Trans A, in press.

[11] Cahn, J. W., and Hilliard, J. E. 1958 Free energy of a nonuniform system. I. Interfacial free energy. J. Chem. Phys. 28, pp. 258-267.

[12] Cahn, J. W., and Hoffman, D. W. 1974 A vector thermodynamics for anisotropic surfaces II. Curved and facetted surfaces. Acta Metall. Mater. 22, pp. 1205-1214.

[13] Carr, J., Gurtin, M. E., and Slemrod, M. 1985 Structured phase transitions on a finite interval. J. Elast. 15, pp. 133-142.

[14] Davis, H. T., and Scriven, L. E. 1982 Stress and structure in fluid interfaces. Advances Chem. Phys. 49, pp. 358-454.

[15] Evans, R. 1979 The nature of the liquid-vapour interface and other topics in the statistical mechanics of non-uniform, classical fluids. Adv. Phys. 28, pp. 143-200.

[16] Fife, P. C. 1995 Private communication.

[17] Ginzburg, V. L., and Landau, L. D. 1950 On the theory of superconductivity. Soviet Phys. JETP 20, p. 1064. 
[18] Glicksman, M. E., and Marsh, S. P. 1993 The Dendrite. In the Handbook of Crystal Growth (ed. D. T. J. Hurle), pp. 1077-1122. Amsterdam: North Holland.

[19] Goldstein, Herbert. Classical Mechanics (2nd ed.) (Addison-Wesley, New York, 1980), Chapter 12.

[20] Halperin, B. I, Hohenberg, P. C., and Ma, S.-K. 1974 Renormalization-group methods for critical dynamics: I. Recursion relations and effects of energy conservation. Phys. Rev. B 10, pp. 139-153.

[21] Hoffman, D. W., and Cahn, J. W. 1972 A vector thermodynamics for anisotropic surfaces I. Fundamentals and application to plane surface junctions. Surface Science 31, pp. 368-388.

[22] Jackson, J. D, Classical Electrodynamics, 2nd Ed. (John Wiley, New York, 1975) p 239.

[23] Karma, Alain and Rappel, Wouter-Jan 1996 Phase-field method for computationally efficient modeling of solidification with arbitrary interface kinetics, submitted to Phys. Rev. Lett.

[24] Kobayashi, R. 1993 Modeling and numerical simulations of dendritic crystal growth. Physica D 63, pp. 410-423.

[25] Lai, Z.-W. 1990 Theory of ordering dynamics for $\mathrm{Cu}_{3}$ Au. Phys. Rev. B 41, pp. 92399256.

[26] Landau, L. D. 1937 On the theory of phase transitions. Reprinted in Men of Physics: L. D. Landau, Vol. 2: Thermodynamics, Plasma Physics, and Quantum mechanics. (ed. D. ter Haar), pp. 61-84. London: Pergamon.

[27] Langer, J. S. 1986 Models of pattern formation in first-order phase transitions. In $D i$ rections in Condensed Matter Physics, (ed. G. Grinstein and G. Mazenko), pp. 164-186. Singapore: World Science Publishers.

[28] McFadden, G. B., Wheeler, A. A., Braun, R. J., Coriell, S. R., and Sekerka, R. F. 1993 Phase-field models for anisotropic interfaces. Phys. Rev. E 48, pp. 2016-2024.

[29] McFadden, G. B., and Wheeler, A. A. 1995 unpublished research.

[30] Murray, B. T., Boettinger, W. J., McFadden, G. B., and Wheeler, A. A. 1994 Computation of dendritic solidification using a phase-field model. In Heat transfer in melting, solidification and crystal growth (ed. I. S. Habib and S. Thynell) pp. 67-76. New York: The American Society of Mechanical Engineers.

[31] Novick-Cohen, A. and Cahn, J. W. 1996 Limiting motion for an Allen-Cahn/CahnHilliard system. Preprint.

[32] Owen, N. C., Rubinstein, J., and Sternberg, P. 1990 Minimizers and gradient flows for singularly perturbed bi-stable potentials with a Dirichlet condition. Proc. Roy. Soc. Lond. A 429 pp. 505-532. 
[33] Oxtoby, D. W. 1991 Crystallization of liquids: a density functional approach. In Liquids, Freezing and Glass Transition (ed. J. P. Hansen, D. Levesque, and J. Zinn-Justin), (Les Houches, Session LI, 1989) pp. 147-191. Amsterdam: Elsevier Science Publisher.

[34] Penrose, O. and Fife, P.C. 1990 Thermodynamically consistent models of phase-field type for the kinetics of phase transitions, Physica D 43 pp. 44-62.

[35] Lord Rayleigh 1892 On the theory of surface forces.-II. Compressible fluids. Phil. Mag. 33, pp. 209-220.

[36] Stanley, H. E. 1971 Introduction to Phase Transitions and Critical Phenomena, Oxford: Oxford University Press.

[37] Taylor, J. E. 1992 Mean curvature and weighted mean curvature, Acta Metall. Mater. 40, pp. 1475-1485.

[38] Taylor, J. E., and Cahn, J. W. 1994 Linking anisotropic sharp and diffuse surface motion laws by gradient flows. J. Stat. Phys. 77, pp. 183-197.

[39] Taylor, J. E. and Cahn, J. W. 1996 Diffuse interfaces with sharp corners and facets: phase field modeling of strongly anisotropic surfaces, preprint.

[40] Truesdell, C., and Noll, W. 1965 The non-linear field theories of mechanics. Vol. III/3 Encyclopedia of Physics, ed. S. Flügge (Springer Verlag, Berlin), pp. 513-515.

[41] Wang, S.-L., Sekerka, R. F., Wheeler, A. A., Murray, B. T., Coriell, S. R., Braun, R. J., and McFadden, G. B. 1993 Thermodynamically-consistent phase-field models for solidification, Physica D 69, pp. 189-200.

[42] Warren, J. A. and Boettinger, W. J. 1995 Prediction of dendritic growth and microsegregation patterns in a binary alloy using the phase-field method. Acta metall. mater. in press.

[43] Wheeler, A. A., Murray, B. T., and Schaefer, R. J. 1993 Computation of dendrites using a phase field model. Physica D 66, pp. 243-262.

[44] Wheeler, A. A. and McFadden, G. B. 1996 A $\xi$-vector formulation of anisotropic phasefield models: 3-d asymptotics. Eur. J. Appl. Maths. in press.

[45] Wheeler, A. A., McFadden, G. B., and Boettinger, W. J. 1996 Phase-field model for solidification of a eutectic alloy. 1996 Proc. Roy. Soc. Lond. A 452, pp. 495-525.

[46] van der Waals, J. D. 1893 Verhandel. Konink. Akad. Weten. Amsterdam (Sect. 1); see Rowlinson, J. S. 1979 Translation of J. D. van der Waals' "The thermodynamic theory of capillarity under the hypothesis of a continuous variation of density." J. Stat. Phys. 20, pp. 197-244. 


